\title{
Embolización de fístula carótido cavernosa indirecta a través de la vena oftálmica superior
}

\author{
J.F. Alén; J. Campollo; J.J. Rivas; A. Lagares; B. Pascual; L. Jiménez-Roldán y R.D. Lobato
}

Servicio de Neurocirugía. Hospital 12 de Octubre. Madrid.

\section{Resumen}

Las fístulas carótido cevernosas (FCC) indirectas o durales son comunicaciones entre el seno cavernoso y ramas extradurales de la arteria carótida interna, la carótida externa o ambas. La mayoría de las FCC indirectas son idiopáticas y aparecen espontáneamente. Los síntomas pueden variar desde una leve inyección conjuntival y escleral hasta la reducción severa de la agudeza visual. En casos poco sintomáticos y sin drenaje leptomeníngeo, el tratamiento conservador estaría indicado inicialmente, pues existe la posibilidad del cierre espontáneo de la fístula. Cuando la sintomatología progresa, requieren algún tipo de tratamiento para cerrarla. Hoy en día, el tratamiento endovascular es el de elección, y la vía transvenosa, con sellado o empaquetado del SC, ha demostrado mayor efectividad que la transarterial. En la mayoría de los casos se puede acceder al SC a través del seno petroso inferior. Sin embargo, en ocasiones no es posible el acceso a la FCC por vía venosa transfemoral, siendo necesario un abordaje directo a la vena oftálmica superior (VOS). Presentamos un caso de FCC indirecta tratada mediante abordaje directo a la VOS y embolización del seno cavernoso.

PALABRAS CLAVE: Fístula carótido cavernosa. Fístula dural. Vena oftálmica superior.

Embolization of indirect carotid-cavernous sinus fistula through the ophthalmic vein

\section{Summary}

Indirect or dural carotid cavernous fistulas are abnormal connections between the cavernous sinus and meningeal branches of the external and/or internal carotid arteries. Most of them are idiopathic and occurs spontaneously. Symptoms vary from a tiny episcleral

Recibido: 30-10-07Aceptado: 25-01-08 injection to a severe visual loss. Conservative therapy is recomended in cases with few symptoms and no leptomeningeal drainage, as spontaneous resolution is not infrequent. Whenever symptoms worsen, treatment of the fistula should be prescribed. Nowadays, transvenous endovascular treatment consisting of packing the cavernous sinus is the first choice. In most cases, cavernous sinus can be approached through the inferior petrosal sinus. However, sometimes that is not possible, and an approach directly through the superior ophthalmic vein could be necessary. We report a case of a patient with a dural carotid cavernous fistula treated with embolization of the cavernous sinus through the ophthalmic vein.

KEYWORDS: Carotid cavernous fistula. Dural fistula. Superior ophthalmic vein.

\section{Introducción}

Las fístulas carótido cavernosas (FCC) son comunicaciones espontáneas o adquiridas entre la arteria carótida y el seno cavernoso que pueden ser clasificadas en directas o indirectas. Las primeras consisten en comunicaciones directas entre la porción cavernosa de la arteria carótida interna y el seno cavernoso (SC). La mayoría son traumáticas o por ruptura de aneurismas de la carótida cavernosa. Hemodinámicamente son de alto flujo, a diferencia de las indirectas, que suelen ser de bajo flujo. Las FCC indirectas o durales son comunicaciones entre el SC y ramas extradurales de la arteria carótida interna, la carótida externa o ambas. La mayoría de las FCC indirectas son idiopáticas y aparecen espontáneamente ${ }^{3}$. En general, las FCC producen signos y síntomas oculares como quemosis, inyección conjuntival, hinchazón palpebral o diplopia, y en casos graves pueden producir proptosis intensa y pérdida de visión.

La mayoría de las FCC directas pueden cerrarse mediante tratamiento endovascular, generalmente por

Abreviaturas. FCC: fistula carótido cavernosa. VOS: vena oftálmica superior. SC: seno cavernoso 


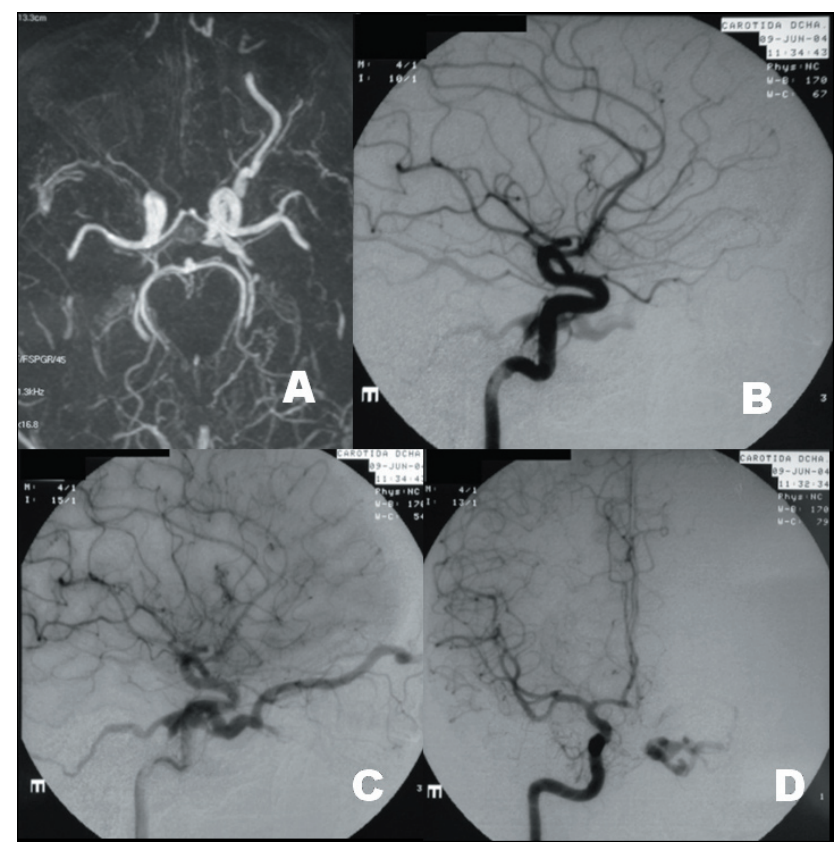

Figura 1. A: Imagen de angio-RM en la que se aprecia el ensanchamiento del seno cavernoso izquierdo y la dilatación de la vena oftálmica de ese mismo lado. B, $C$ y D: Arteriografía cerebral en proyección lateral y anteroposterior de carótida derecha que es por donde se rellena la fistula, la cual drena fundamentalmente a través de la vena oftálmica superior izquierda dilatada.

vía arterial mediante coils o balones ${ }^{8,17}$, aunque también se han obtenido buenos resultados por vía venosa ${ }^{13}$. El tratamiento de las FCC indirectas o durales es más complicado, siendo la vía venosa de elección para la mayoría de los autores ${ }^{17,12,18,21,26}$. Sin embargo, en ocasiones no es posible el acceso a la FCC por vía venosa transfemoral, siendo necesario un abordaje directo a la vena oftálmica superior (VOS), que fue descrito en $1969^{19}$ y posteriormente utilizado por diferentes autores para el tratamiento de $\mathrm{FCC}^{2,10,15,18,21}$.

Presentamos un caso de FCC indirecta tratada mediante abordaje directo a la VOS y embolización del seno cavernoso.

\section{Caso clínico}

Se trata de una mujer de 53 años intervenida de cataratas, que consultó por un cuadro de 18 meses de evolución de una leve proptosis progresiva del ojo izquierdo y edema palpebral que no se acompañaban de pérdida subjetiva de la agudeza visual. Refería también acúfenos pulsátiles en el oído izquierdo. En la exploración destacaba una proptosis e inyección conjuntival en el ojo izquierdo sin alteraciones en la motilidad ocular. La agudeza, campimetría y presión

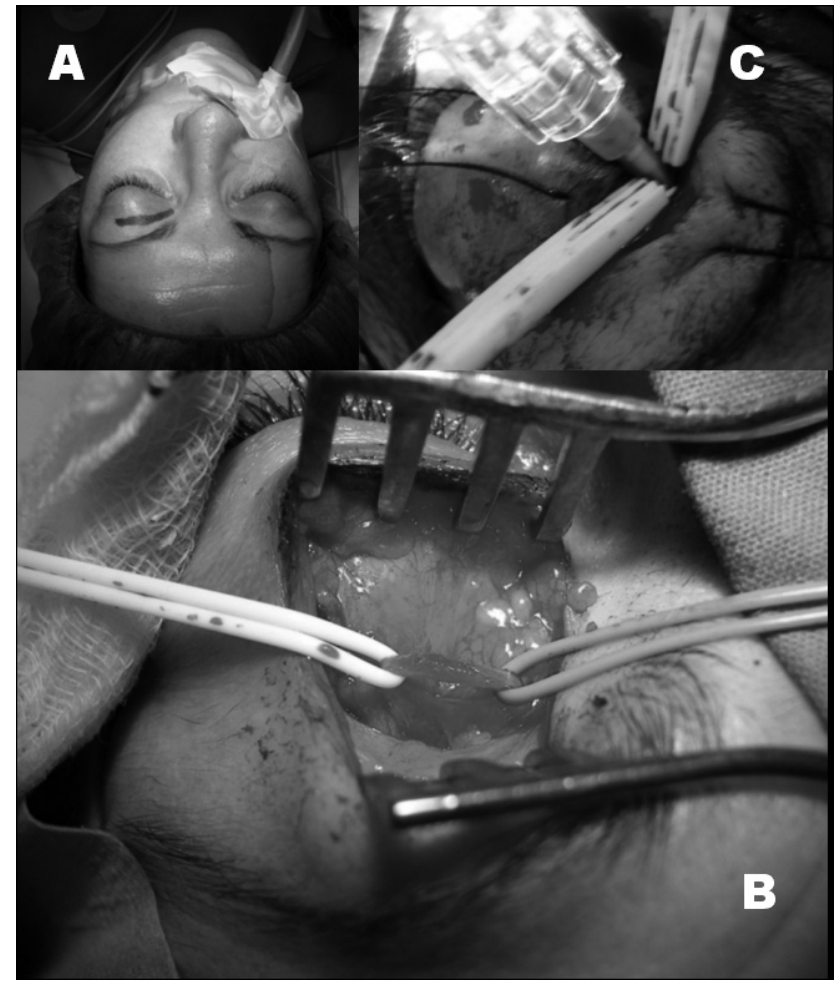

Figura 2. Fotografias del procedimiento quirúrgico: $A$ : Incisión realizada en el párpado; B: Vena oftálmica disecada antes de realizar la venotomía; $C$ : Abocath introducido por la vena oftálmica, a través del cual se introduce el microcatéter.

intraoculares eran normales. En el estudio mediante angioRM se apreciaba un ensanchamiento del SC izquierdo y dilatación de la VOS izquierda (Figura 1), por lo que se realizó una arteriografía cerebral que mostraba una FCC dural que se llenaba a través de ramas meningohipofisarias y tronco inferolateral de la carótida interna derecha, así como por ramas de la arteria maxilar (Figura 1). Se intentó el tratamiento endovascular transvenoso, intentando acceder al SC a través de seno petroso inferior, que no pudo ser canalizado tras varios intentos, por lo que se optó por el tratamiento conservador. Se adiestró a la paciente para realizar autocompresión de la carótida común derecha, pero tras 4 meses de seguimiento la paciente acudió a urgencias refiriendo episodios autolimitados de visión borrosa y diplopia de aparición reciente. En la exploración neurológica no existían alteraciones relevantes, y la exploración oftalmológica seguía siendo normal. Dada la reciente aparición de síntomas visuales se decidió realizar un tratamiento endovascular mediante abordaje directo de la VOS.

La intervención se llevó a cabo según la técnica descrita por Miller y cols ${ }^{18}$. Bajo anestesia general, en la sala de neurorradiología intervencionista, se realizó abordaje 


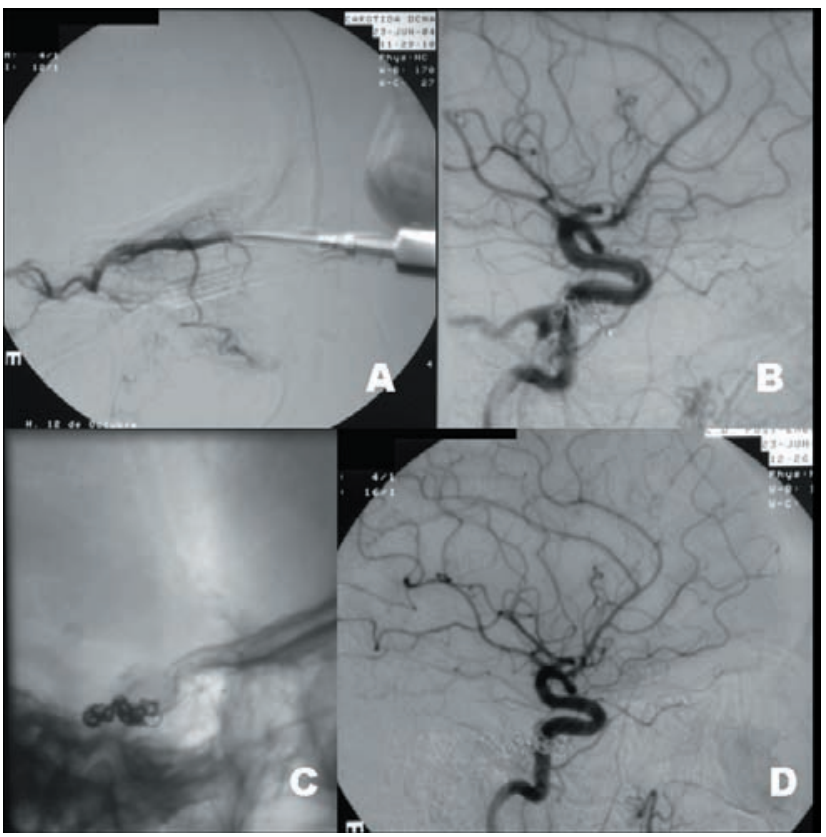

Figura 3. Imágenes del procedimiento endovascular. A: Venograma inicial; B: Inyección en carótida derecha tras la colocación de 3 coils, con relleno de FCC y drenaje a seno petroso inferior; $C$ : Imagen final sin substracción tras la colocación de 7 coils; D: Inyección en carótida derecha final tras la embolización.

transpalpebral hasta localizar la VOS dilatada (Figura 2) con ayuda de lupas de aumento. Se canalizó la VOS con un "abbocath" (16G Protectiv ${ }^{\circledR}$ Plus) para introducir a través de él un microcatéter (Excelsior ${ }^{\circledR}$, Boston Scientific Corporation) hasta el SC izquierdo. Una vez comprobada la adecuada colocación del microcatéter se rellenó el SC con 7 coils (Matrix ${ }^{\circledR}$ Detachable Coils, Boston Scientific Corporation) (Figura 3). Además, se colocó un introductor en la arteria femoral para realizar los controles tras la colocación de los coils, mostrando el estudio angiográfico final la ausencia de relleno de la FCC (Figura 3).

Tres meses después habían desaparecido la proptosis, la inyección conjuntival y los acúfenos, y la paciente no refería déficit visual alguno ni diplopia. El resultado estético fue muy bueno, sin apreciarse la cicatriz palpebral a las pocas semanas de la intervención. El estudio mediante angio-RM y la angiografía de control a los 11 meses mostraban la ausencia de relleno de la FCC (Figura 4).

\section{Discusión}

Las FCC durales o indirectas suelen ser de bajo flujo, y por lo general presentan gran cantidad de finas arterias aferentes que pueden provenir de ramas durales de la carótida interna contralateral e incluso de la arteria ver-

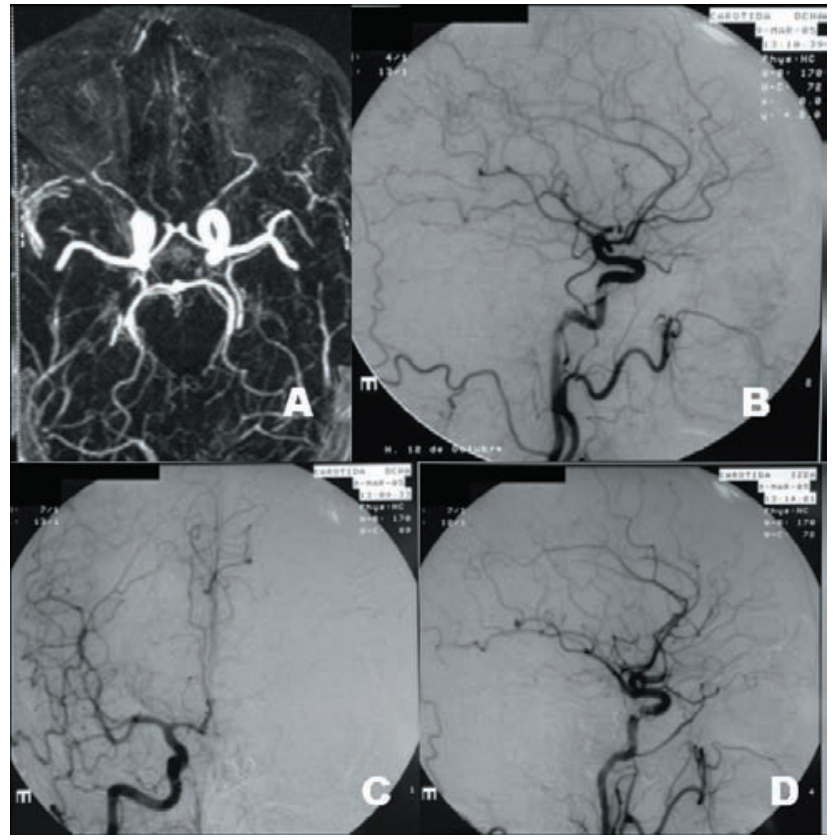

Figura 4. Control a los 11 meses. A: Angio-RM donde no se aprecia ni ensanchamiento del seno cavernoso ni dilatación de la vena oftálmica. B y C: Imágenes de angiografía en proyección lateral y anteroposterior de la carótida derecha común y D: imagen de angiografía en proyección lateral de la carótida izquierda común. Se aprecia la oclusión completa de la FCC.

tebral. Los síntomas pueden variar desde una leve inyección conjuntival y escleral hasta la reducción severa de la agudeza visual. La afectación visual suele producirse por el glaucoma asociado a la hipertensión venosa, aunque también por la diplopia, la proptosis con exposición de la córnea, el edema macular, la retinopatía o por isquemia del nervio óptico ${ }^{10,11,15}$. Raramente suponen un riesgo vital para el paciente, existiendo riesgo de sangrado intracraneal únicamente en los casos que presentan un drenaje venoso retrógrado leptomeníngeo ${ }^{16}$.

En casos poco sintomáticos y sin drenaje leptomeníngeo, el tratamiento conservador estaría indicado inicialmente, pues existe la posibilidad del cierre espontáneo de la fístula

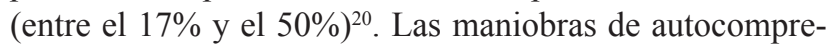
sión manual de la carótida común pueden conseguir la curación por trombosis en casos de muy bajo flujo ${ }^{1}$, y se ha descrito también su cierre tras la práctica de angiografía cerebral diagnóstica ${ }^{9}$. Sin embargo, en pacientes con alto riesgo de ictus embólico o en pacientes predispuestos a síncopes vasovagales por hipotensión, no debería aconsejarse este tratamiento ${ }^{21}$.

Cuando la sintomatología progresa (pérdida de visión, glaucoma, etc.), requieren algún tipo de tratamiento para cerrar la fístula, y esto es prácticamente necesario cuando 
la clínica es bilateral. El exoftalmos bilateral no siempre implica la presencia de dos fístulas independientes sino la existencia de una comunicación entre ambos senos cavernosos a través del seno coronario 9 . En el pasado, el tratamiento quirúrgico era el más empleado, generalmente con sacrificio de la carótida responsable mediante ligadura o "trapping", pero la morbilidad era alta y las recurrencias eran frecuentes por recanalizaciones de colaterales de la vertebral y de la arteria oftálmica ${ }^{24}$.

Hoy en día, el tratamiento endovascular de las FCC durales es el de elección, y la vía transvenosa, con sellado o empaquetado del SC, ha demostrado mayor efectividad que la transarterial ${ }^{12,16,26}$. En la mayoría de los casos se puede acceder al SC a través del seno petroso inferior, aunque en ocasiones esto no es posible por imposibilidad de canalizarlo o por estar trombosado ${ }^{12,21,26}$. En caso de no poder acceder por dicha vía existen otras alternativas que incluyen el acceso a través del plexo venoso del clivus ${ }^{7}$, el plexo pterigoideo ${ }^{14} \mathrm{o}$ incluso a través de venas corticales ${ }^{4}$. Sin embargo, estas vías tampoco son siempre practicables endovascularmente. Por otro lado, si el drenaje es mayoritariamente anterior, la embolización a través de la VOS es un tratamiento eficaz en la mayoría de las ocasiones. Desde el punto de vista anatómico la VOS drena inferiormente a la vena angular y la vena facial común, la cual está conectada a las venas yugulares interna y externa. En sentido superior, la VOS se continúa con la vena nasofrontal, la vena temporal medial y la retromandibular, la cual drena a la vena facial común. En pacientes con FCC durales, estos canales venosos suelen estar dilatados, al ser vías de drenaje de la fístula, por lo que pueden servir para acceder al SC endovascularmente. No obstante, en muchas ocasiones existen zonas muy estrechas y tortuosas para poder navegar de manera adecuada con microcatéteres y se puede hacer preciso canalizar de manera directa la VOS, como ocurrió en nuestro caso. Algunos autores han optado por la punción directa de la $\operatorname{VOS}^{5,22}$ o la canalización de la VOS a través del abordaje directo a una vena frontal ${ }^{25}$.

$\mathrm{El}$ abordaje directo a la VOS no es un tratamiento nuevo para las FCC. Diferentes autores han reportado casos aislados o pequeñas series con buenos resultados ${ }^{1,2,10,15,18,21}$. En nuestro caso no encontramos otra forma de acceso al SC y optamos por esta vía, que nos permitió un punto de apoyo cercano al SC para poder empaquetarlo con coils. La presencia de una VOS dilatada facilita el abordaje directo, mientras que en los casos en que la VOS no está muy dilatada o está trombosada, esta tarea puede ser más difícil de conseguir. Los materiales utilizados para el empaquetado del SC han sido los coils y los balones, reportándose resultados similares con ambos materiales ${ }^{18}$. Algunos autores han utilizado cianoacrilato (NBCA) combinado con coils para sellar por completo las fístulas ${ }^{6,23}$. Nosotros decidimos utilizar los coils, siguiendo las recomendaciones de
Quiñones y cols, por la mayor facilidad de manejo y por la posibilidad de utilizar un microcatéter más fino ${ }^{21}$.

Las potenciales complicaciones de este tratamiento son el sangrado abundante por ruptura de la vena oftálmica, el daño a la tróclea o a otras estructuras intraorbitarias o la infección. Sin embargo, estas complicaciones generalmente son motivadas por intentos de canalización de venas trombosadas o no dilatadas ${ }^{10}$.

Consideramos que los diferentes trabajos en la literatura demuestran la efectividad y la seguridad de este tratamiento, y en nuestro caso seleccionado corroboramos la efectividad y seguridad del mismo. Sin embargo, este tratamiento no está exento de posibles complicaciones por lo que pensamos que debe indicarse únicamente en casos con imposibilidad de canalizar el seno cavernoso a través del seno petroso inferior y en pacientes con dilatación sin trombosis de la vena oftálmica superior.

\section{Biblografía}

1. Annesley-Williams, D.J., Goddard, A.J., Brennan, R.P., Gholkar, A.: Endovascular approach to treatment of indirect carotico-cavernous fistulae. Br. J Neurosurg. 2001; 15: 228-233.

2. Baldauf, J., Spuler, A., Hoch, H.H., Molsen, H.P., Kiwit, J.C., Synowitz, M.: Embolization of indirect carotid-cavernous sinus fistulas using the superior ophthalmic vein approach. Acta Neurol. Scand. 2004; 110: 200-204.

3. Barrow, D.L., Spector, R.H., Braun, I.F., Landman, J.A., Tindall, S.C., Tindall, G.T.: Classification and treatment of spontaneous carotid-cavernous sinus fistulas. J Neurosurg. 1985; 62: 248-256.

4. Bellon, R.J., Liu, A.Y., Adler, J.R., Jr., Norbash, A.M.: Percutaneous transfemoral embolization of an indirect carotid-cavernous fistula with cortical venous access to the cavernous sinus. Case report. J Neurosurg. 1999; 90 : 959-963.

5. Benndorf, G., Bender, A., Campi, A., Menneking, H., Lanksch, W.R.: Treatment of a cavernous sinus dural arteriovenous fistula by deep orbital puncture of the superior ophthalmic vein. Neuroradiology 2001; 43: 499-502.

6. Berkmen, T., Troffkin, N.A., Wakhloo, A.K.: Transvenous sonographically guided percutaneous access for treatment of an indirect carotid cavernous fistula. AJNR Am J Neuroradiol. 2003; 24: 1548-1551.

7. Cheng, K.M., Chan, C.M., Cheung, Y.L.: Transvenous embolisation of dural carotid-cavernous fistulas by multiple venous routes: a series of 27 cases. Acta Neurochir. (Wien. ) 2003; 145: 17-29.

8. Debrun, G., Lacour, P., Vinuela, F., Fox, A., Drake, C.G., Caron, J.P.: Treatment of 54 traumatic carotid-cavernous fistulas. J Neurosurg. 1981; 55: 678-692.

9. Díez Lobato, R., Escudero, L., Lamas, E.: Bilateral 
dural arteriovenous fistula in the region of the cavernous sinus. Neuroradiology 1978; 15: 39-43.

10. Goldberg, R.A., Goldey, S.H., Duckwiler, G., Vinuela, F.: Management of cavernous sinus-dural fistulas. Indications and techniques for primary embolization via the superior ophthalmic vein. Arch. Ophthalmol. 1996; 114 :707-714.

11. Goto, K., Hieshima, G.B., Higashida, R.T., et al.: Treatment of direct carotid cavernous sinus fistulae. Various therapeutic approaches and results in 148 cases. Acta Radiol. Suppl 1986; 369: 576-579.

12. Halbach, V.V., Higashida, R.T., Hieshima, G.B., Hardin, C.W., Pribram, H.: Transvenous embolization of dural fistulas involving the cavernous sinus. AJNR Am J Neuroradiol. 1989; 10: 377-383.

13. Halbach, V.V., Higashida, R.T., Hieshima, G.B., Hardin, C.W., Yang, P.J.: Transvenous embolization of direct carotid cavernous fistulas. AJNR Am J Neuroradiol. 1988; 9: 741-747.

14. Jahan, R., Gobin, Y.P., Glenn, B., Duckwiler, G.R., Vinuela, F.: Transvenous embolization of a dural arteriovenous fistula of the cavernous sinus through the contralateral pterygoid plexus. Neuroradiology 1998; 40: 189-193.

15. Klink, T., Hofmann, E., Lieb, W.: Transvenous embolization of carotid cavernous fistulas via the superior ophthalmic vein. Graefes Arch. Clin. Exp. Ophthalmol. 2001; 239: 583-588.

16. Klisch, J., Huppertz, H.J., Spetzger, U., Hetzel, A., Seeger, W., Schumacher, M.: Transvenous treatment of carotid cavernous and dural arteriovenous fistulae: results for 31 patients and review of the literature. Neurosurgery 2003; 53: 836-856.

17. Lewis, A.I., Tomsick, T.A., Tew, J.M.J.: Management of 100 consecutive direct carotid-cavernous fistulas: results of treatment with detachable balloons. Neurosurgery $1995 ; 36$ :

18. Miller, N.R., Monsein, L.H., Debrun, G.M., Tamargo, R.J., Nauta, H.J.: Treatment of carotid-cavernous sinus fistulas using a superior ophthalmic vein approach. J Neurosurg. 1995; 83: 838-842.

19. Peterson, W., Valbert, J., Whittingham, D.: Electrically induced thrombosis of the cavernous sinus in the treatment of carotid cavernous fistula. In: Proceedings of the fourth international congress of neurological surgery. 193, 105. 1969.

20. Phelps, C.D., Thompson, H.S., Ossoinig, K.C.: The diagnosis and prognosis of atypical carotid-cavernous fistula (red-eyed shunt syndrome). Am J Ophthalmol. 1982; 93: 423-436.

21. Quinones, D., Duckwiler, G., Gobin, P.Y., Goldberg, R.A., Vinuela, F.: Embolization of dural cavernous fistulas via superior ophthalmic vein approach. AJNR Am J Neuroradiol. 1997; 18: 921-928.

22. Teng, M.M., Guo, W.Y., Huang, C.I., Wu, C.C., Chang, T.: Occlusion of arteriovenous malformations of the cavernous sinus via the superior ophthalmic vein. AJNR Am J Neuroradiol. 1988; 9: 539-546.

23. Teng, M.M., Lirng, J.F., Chang, T., et al.: Embolization of carotid cavernous fistula by means of direct puncture through the superior orbital fissure. Radiology 1995; 194: 705-711.

24. Uflacker, R., Lima, S., Ribas, G.C., Piske, R.L.: Carotid-cavernous fistulas: embolization through the superior ophthalmic vein approach. Radiology 1986; 159: 175179.

25. Venturi, C., Bracco, S., Cerase, A., et al.: Endovascular treatment of a cavernous sinus dural arteriovenous fistula by transvenous embolisation through the superior ophthalmic vein via cannulation of a frontal vein. Neuroradiology 2003; 45: 574-578.

26. Yu, S.C., Cheng, H.K., Wong, G.K., Chan, C.M., Cheung, J.Y., Poon, W.S.: Transvenous embolization of dural carotid-cavernous fistulae with transfacial catheterization through the superior ophthalmic vein. Neurosurgery 2007; 60: 1032-1037.

Alén, J.F.; Campollo, J.; Rivas, J.J.; Lagares, A.; Pascual, B.; Jiménez-Roldán, L.; Lobato, R.D.: Embolización de fístula carótido cavernosa indirecta a través de la vena oftálmica superior. Neurocirugía 2008; 19: 338-342.

Correspondencia postal: Dr. José A Fernández Alén. Servicio de Neurocirugía. Hospital 12 de Octubre. Avda Andalucía s/n. 28041 Madrid 